\title{
Perception and Text Comprehension. It's a Matter of Perception!
}

\author{
https://doi.org/10.3991/ijet.v13i07.7909 \\ Aglaia Tourimpampa, Athanasios Drigas ${ }^{(\varpi)}$ \\ N.C.S.R. 'Demokritos', Athens, Greece \\ dreit.demokritos.gr \\ Alexandra Economou, Petros Roussos \\ University of Athens, Athens, Greece
}

\begin{abstract}
This study is a comprehensive attempt to assess the impact of the cognitive skill of perception in the ability to comprehend a text. More specifically, it investigates the function of perception as a primary structure of the human brain to contact the world and examines the certain cognitive processes of perception that affect text comprehension. It is also presented the relation between cognitive perception and the linguistic approach of pragmatics in order the subject to comprehend the text. Perception is the organization, identification and interpretation of sensory information in order to represent and understand the environment. Pragmatics is the linguistic field that studies how people comprehend and produce speech or a text as a communicative act. Furthermore, it features the current scientific achievements on the ICTs processes and tools, which exploit the assessment of perception in text comprehension.
\end{abstract}

Keywords-perception, text comprehension, pragmatics, ICTs in text and reading comprehension

\section{Introduction}

The cognitive ability of human to understand the world and its structure relies on the ability of perception. Human brain accepts all the sensory information from the environment and then by processing this information in order to classify and organize it, it forms the perception of the world [1]. The nervous system is responsible for the intake of the sensory information. Perception is also directly related to the cognitive abilities of memory, observation, expectation and attention and can be divided into two processes as well. The function of perception begins firstly with the sensory data input from external environment and then it interprets the stimulus into an information that makes sense such as the shape for object recognition. The second part of the process moves forward according to the influence of a person's knowledge and the development of his other cognitive abilities (attention, memory etc.)

But, as perception seems to be a more complicated function of the nervous system, there has been a great interest of various scientific fields to study the ability of percep- 
tion. Psychophysics explores the physical sensory input of perception while neuroscience investigates the mental processes of perception. Although the sensory stimuli were considered as the only real existing objects, mental illusions and ambiguous images lead the science of philosophy to study how much perception is related either to the objective reality or the human brain.

Human brain's function is based on perceptual systems that enable individuals to understand the world. They are structured in a modular way with different areas of processing and some of them are formed as sensory maps that chart concepts of the world. Due to this, Jerome Bruner developed a perception model and according to it, people, in order to form a specific opinion when they encounter an unfamiliar target, they try to collect information and gradually familiarize themselves with the subject by boxing the analysis into different categories. Secondly, according to Saks and Jones, perception is tri-dimensional. Three are factors affect perception which are the Perceiver, the Target and the Situation. The Perceiver has a concrete experience, motivational and emotional state in order to understand the Target (the person who is being perceived). Thirdly, the Situation plays a great role in the process of perception because various situations offer additional information about the target perception.

Furthermore, perception disposes some features that describe more specifically the way perception functions. Perceptual constancy is the ability of perception to recognize objects from different sensory data. The principle of grouping is another feature that presents the natural ability of human mind to perceive objects as organized patterns and objects. Moreover, another parameter of perception is the contrast effects according to which perception of an object may be influenced by its context. Additionally, factors such as experience of the individual can lead him to make more concrete perceptional concepts while, motivation and expectation of him as well, can result varying perception of ambiguous figures or concepts [2].

This study is also intended to investigate the relation between perception and the linguistic field of pragmatics in order to identify the interaction between the two factors and how these affect text comprehension. Pragmatics study the way individuals perceive and produce speech in certain communicative circumstances. In every communicative act there are two intentions of the individual: The informative one and the communicative one. Should the subject is capable of understanding social, cultural and in general all pragmatic knowledge of the communicative act, obtains the pragmatic competence. Certain factors that are studied in pragmatics are deixis, the feature that refers to the context of the concept, presupposition, referring to the logical meaning of a sentence, performance of the speaker, meaning all the acts that the communicative message includes and implicature, referring to the implications included in a text arising from its context [3].

The paper also indicates the impact of several other factors of cognitive perception that affect text comprehension. Mental models and situation models structured in human brain affect the perception of a text as well as causal thinking or conscious and unconscious perception which an approach to the relations between phenomenal experience and perceptual processes. It is also investigated how parsing a narrative into separate events impair anaphor resolution. In general it is examined how event segmentation affect perception of text. Moreover, there is an effort of understanding the 
process that human brain does in order to match the pronoun with its antecedent. It also studied the interaction of contextual information during reading, the emotional perception, the background knowledge of the individual in order to perceive the text and the basic gestalt principles referring to the perception of the narrative text.

\section{Perception and Text Comprehension}

In this chapter will be investigated the cognitive parameters and brain processes of perception which can be associated with text comprehension. One of these parameters is the form of situation models and it is illustrated in the study of Rolf A. Zwaanis. A situation model is formed by the human brain in order to comprehend a discourse or a text and it is based on the development of mental representations in relation with the state of affairs implied by the text [4].

In the study of D. Morrow, S. Greenspan and G. Bower, it was attempted an investigation on how the subject can have access to the information of situation models during narrative comprehensionIn the three-part experiment, researchers, administered to the subjects a diagram of a building to memorize it and then read narratives describing the moves of a person inside a building in order to accomplish a target. To investigate accessibility, the narrative was interrupted by demonstrating the names of two objects to the subjects in order to decide if the objects are placed in the same or different rooms of the building. Experiment 1 examined objects' accessibility after goal utterances. The most accessible ones were those situated in the goal room. In Experiment 2, the accessibility of the information was based on the location of the protagonist in the situation model, while in Experiment 3 indicated that after the path sentences mentioned in the narrative, where the protagonist was heading for the goal, both path and goal rooms were accessible. So, it is inferred that the information accessibility has to do more with the situations described situations rather than the outward structure of the narrative [5].

The same research assumption is presented in Rolf A. Zwaan, Mark C. Langston, Arthur C. Graesser. They describe how readers create situations models based on the events taking place in the text, constructing this way an event indexing model. Events are the basic points of the situation model created in a narrative, connected to the dimensions of time, space, protagonist, causality and intentionality [6].

According to the findings of a similar experiment, attempted by Arthur M. Glenberg, Marion Meyer and Karen Lindem, mental models represent the general perception if the text, not just the features of the text itself and that's why they play a primary role in text comprehension. Subjects read texts in which there different described events-the ones in which the actor is associated with the target object and the ones in which the actor is dissociated with the target object. Experiment results indicated that the target object remained foregrounded when the text described events in which the protagonist and the object were spatially associated [7].

Another crucial parameter which is related to text comprehension is conscious and unconscious perception, exposed in the study of Antony J. Marcel. More specifically, it presents the cognitive function of consciousness and non conscious perceptual pro- 
cesses and their relation. It is claimed that sensory data are not identically represented by the perceptual procedures. Non conscious perceptual processes offer a high level explication of phenomenal experience, in any other possible cognitive form whereas conscious perception is a cognitive function which tries to define as much data as possible in the most effective way[8].

In the same scientific field, A. Marcel carries out five experiments in order to investigate the relation of masking to consciousness and visual word processing. In experiments 1 and 2, subjects were given a single word or blank field followed by a pattern mask and they were asked to decide whether anything preceded the mask, and to which of the two probe words

was what preceded the mask more similar graphically or semantically. In the third experiment, subjects were administered a task in which they had to make a manual recognition of color patches which were either preceded by masked words so as to prevent awareness. Color compatible words made reaction time easier whereas color incongruent words made it more difficult. In experiment 4, was attempted a word task decision. Subjects were administered a critical letter string following another not requiring a response. Both words were either semantically correlated or not. The first letter was either masked or unmasked. In both cases, the correlation result was equal. The correlation effect on a subsequent lexical decision was repeated on experiment 5 as well [9].

Two experiments, made by Danielle S. McNamara \& Walter Kintsch, examined the results of background knowledge on learning form high and low coherence history texts. Specifically, in the first experiment, subjects were given exercises of free recall, multiple-choice questions, and a keyword sorting task. It turned out that high knowledge readers completed the tasks successfully even after reading a low coherence text. Experiment 2, comprised of the same tasks indicated the same results [10].

In the paper of Daniel G. Morrow, it is supported the idea that readers can perceive a narrative by creating a coherent representation structure. Based on this, experiments showed that properties of the characters and events expressed by narratives are often more important for guiding referent assignment than the order in which these parts of narratives are mentioned. The results suggest that readers associate information about the characters, events, and places that narratives express with information about their order of mention in order to attach referents and that this process is part of the procedure of constructing a structure that represents the world described by the narrative[11].

Paul van den Broek presents the theory of causal inference making that considers the differences among properties of causality. This paper attempts to depict the main properties of causal relations in texts. It also describes the cognitive process $\mathrm{s}$ which use these properties in order to organize the causal information of the text. This causal schema of the events in the text can lead to all causal assumptions and it can also delimit the content of the inferences [12].

A significant point of the cognitive processes as far as concerns text comprehension is the coherence of the text. In the paper of Ted J. M. Sanders \& Leo G. M. Noordman there is focus on the cognitive status of coherence relations of the text. In an experiment using reading, verification, and free recall tasks, 2 critical points of the 
structure of expository texts were examined: the type of coherence relation between segments (problem-solution vs. list) and the linguistic marking of the relations by means of signaling phrases (implicit vs. explicit). The results indicated that both factors affected text processing [13].

In their study, Ekaterina P.Volkova, Betty J.Mohler, Detmar Meurers, Dale Gerdemann, Heinrich H. Bulthoff propose an Emotion Analysis System which will be assigned to text units. In this paper also, researchers carry out an experiment presenting how an annotation task can be organised so that the subjects perform emotion analysis using a rich set of emotion categories. This procedure brings Emotion Analysis closer to actual human emotional perception of text [14].

Lastly, Ulrich Schiefele attempts a review of the relation between text comprehension and the concept of interest. Interest is defined as a content-specific motivational characteristic composed of feeling-related and value-related capacities. Results from several studies indicate the importance of interest for the depth of text comprehension, the use of learning strategies, and the quality of the emotional experience while learning [15].

\section{$3 \quad$ Perception and Pragmatics}

As it is mentioned above, perception is the cognitive skill of the organization, identification and interpretation of sensory information in order to represent and understand the environment. Pragmatics is the linguistic field that studies how people comprehend and produce speech or a text as a communicative act. In this chapter, there is an attempt to examine some concepts of pragmatics, how they are related to perception and in what is their impact in text comprehension.

Douglas Vipond and Russel A. Hunt illustrate in their paper the so called "pointdriven" reading. They argue that readers try to understand a text or an oral conversation by finding out what is the main idea of the narrator. Their understanding is pointdriven in this sense.

Point-driven reading is conceptually different from story-driven and informationdriven types. Each type is related to several cognitive strategies, with point-driven reading, specifically, characterized by coherence, narrative surface, and transactional strategies. Using a modern short story, it is indicated how point-driven readings may vary from other kinds [16].

The paper of Christian Burgersa, Margot van Mulkenb, Peter Jan Schellen, presents two experiments which illustrate that textual characteristics of irony (type of ironic evaluation and irony markers) can affect comprehension, perceived complexity and approaches towards the utterance and text. Outcomes of experiment 1 demonstrate that explicitly evaluative irony is perceived as less complex and is more acknowledged than implicitly evaluative irony. In experiment 2 , irony markers were found to enhance comprehension, reduce perceived complexity and make perspectives towards the utterance more positive. Both experiments also demonstrate that the influence of irony on attitudes depends on comprehension and complexity. If irony is 
understood as relatively easy, it is better liked than when it is not understood as relatively difficult [17].

The connotations of a language expression are pragmatic reactions that arise from encyclopaedic knowledge about its denotation (or reference) and also from experiences, beliefs, and prejudices about the contexts in which the expression is typically used. The connotation of a language expression is apparently distinct from its sense, definition and reference. Connotation is involved enough with notions of appropriateness in language use and obviously with pragmatic effect. In general, connotation is a highly pragmatic category of meaning[18].

Paul Grice in his study presents the method with which logical terms such as some, or and and develop extralogical meanings in conversational contexts and he also used the term implicature to describe the pragmatic inference linking word meanings to speaker's meanings[19].

Darcia Narvaez in her paper points out the matter of moral schema and investigates the differences in this schema development using techniques intermediate between measuring implicit knowledge (such as with the Defining Issues Test) and explicit knowledge (such as with the Moral Judgment Interview). Results demonstrate compelling differences in the comprehension of moral narratives based on age/education and on level of expertise. Also, intended moral themes in stories are not perceived by younger children [20].

Another concept referred to text comprehension concerning pragmatics and perception is the Gestalt imagery, introduced in the study of Nanci Bell. Gestalt imagery is the ability to create imaged wholes - is a significant matter in oral and written language comprehension. Despite good decoding, good vocabulary, and adequate background experiences, many individuals experience weak gestalt imagery, thus processing "parts" rather than "wholes," from verbal stimuli, spoken or written. This may lead to a Language Comprehension Disorder that may appear as symptoms like weak reading comprehension, weak oral language comprehension, weak oral language expression, weak written language expression, difficulty following directions, and a weak sense of humor [21]..

\section{$4 \quad$ ICTs and Text Comprehension}

In the last chapter of this paper there is an exposition of ICTs in text comprehension. More specifically, there is the description of web based, Artificial Intellinenge and mobile tools, devices and computer assisted methods which rely on certain cognitive skills of the human mind in order to comprehend speech or text.

\subsection{Web-Based Applications}

The paper of Lara L. Lomicka investigated the effects of multimedia reading software on reading comprehension. Specifically, the study aimed to investigate the impact of multimedia annotations in the level of comprehension. Twelve college were asked to think aloud during the reading of text on the computer screen. Participants 
read the text in three different ways: full glossing, limited glossing, or no glossing. Moreover, a tracker was installed in the software to record the amount and type of glosses, and length of time that each was consulted. The results indicate an increase in the number of causal inferences created for students who had access to full glossing. As a conclusion, computerized reading with full glossing may develop a deeper level of text comprehension [22].

In the presentation of Kelly Beasly, it is demonstrated the Reader Self-Perception Scale. It is a tool used to measure how intermediate-level (4th through 6th grade) children feel about themselves as readers. The general question gets the children to think about their reading ability. It is a four-facto model that consists of four parts: a) progress (how one's perception of present reading performance compares with their past performance). b)Observational Comparison (deals with how the subject perceives his reading performance to compare with classmates).c) Social Feedback (includes direct and indirect input about reading from teachers, classmates and persons in the family) and d) Physiological States9refers to internal feelings that the subject experiences during reading). These four factors working together, help the teacher understand children's perception[23].

Due to the fact that, on the Common European Framework of Reference for Languages (CEFR) scale, the average reading comprehension level of the Dutch population face difficulties, Eric Velleman and Thea van der Geest, built a free available online tool to give content editors some elements of the CEFR reading comprehension level of their texts. This paper exposes the parameters of the tool and proposes a list of possible extensions to improve the quality and usability of the output[24].

In the following paper of Wen Hao Huang are demonstrated findings of an evaluation of an on line game-based learning environment by examining the learners' motivational and cognitive processing. In the survey participated 144 undergraduate students after their participation in the online available at the Nobel Prize Foundation website teaching the Heckscher Ohlin Theory on international trade. Findings of cognitive load indicated that learners' observed a higher level of intrinsic load that the relevant load because of the new subject matter. The result of the cognitive load pointed out the correlation between learners' motivational and cognitive processing [25].

Another study of Franck Amadieu, André Tricot and Claudette Mariné illuminates the interaction between prior knowledge and concept mapping structure on hypertext comprehension. Comprehension was examined according to three dependent variables: comprehension outcomes, coherence of the reading sequences and feelings of disorientation. The results confirmed most of the assumptions showing an interaction effect on each measure. For low prior knowledge readers, a hierarchical structure improved comprehension skills, and led them to follow coherent reading sequences. Moreover, prior knowledge was correlated with the cognitive requirements of reading non-linear texts. Overall, the study focused on the relation of prior knowledge in hypertext comprehension and pointed out the interest of examining the nature of the online inferences and on relations between performance, navigation and disorientation [26]. 
The goal of Julie Coiro and Elisabeth Dobler in their study, was to investigate the nature of reading comprehension processes while reading on the Internet. Eleven sixth-grade students with the high level reading skills and Internet reading experiences, were selected from a population of 150 sixth graders. These 11 skilled readers met individually with a researcher and completed two separate tasks that involved reading within multilayered websites or using the Yahooligans! search engine. Students answered specific questions about their strategy use in a follow-up interview after each reading session. The procedure consisted of four distinct phases, each of which involved reviewing data from think-aloud protocols, field observations, and semi structured interviews to provide observations on the nature of online reading comprehension. Findings indicated that successful Internet reading demanded both similar and more complex applications of prior knowledge sources, inferential reasoning strategies, as well as self-regulated reading processes[27].

Johannes Naumann suggests that online reading engagement forecasts the engagement in digital reading. Dedication in digital reading according to the model is reflected in task-adaptive navigation, and task-adaptive navigation predicts digital reading performance over and above print reading skill. Moreover, a task-adaptive navigation predicts digital reading performance over and above print reading skill. Linear mixed models were used to predict navigation behavior from the correlation of information and social online reading engagement with navigation needs. High information engagement was related to more task-adaptive navigation behavior. Generalized linear mixed models were used to predict task performance by the interaction of navigation demands and navigation behavior. Adaptive navigation behavior foresaw digital reading task performance, as shown by significant interactions between navigation behavior and navigation demands. These results enhance the proposed model of online reading engagement [28].

Mina C. Johnson Glenberg outlines the function of the metacognitive strategies for text comprehension using web-based tools. The web based application called 3D Readers, was used to train twenty middle school poor reading comprehenders in two metacognitive strategies. The training texts were science-oriented and merged the narrative and expository genres. Subjects answered two main experimental questions: (1) Was the comprehension better after reading experimental texts with embedded verbal (generate questions) and visual (create a model) strategies compared to control texts? (2) Did the embedded strategies affect elective rereading of the texts? The data answered both questions positively. Comprehension, as assessed with constructed answers, was significantly higher in the experimental condition, thus demonstrating the efficacy of training verbal and visual strategies in a Web-based environment[29].

The study of Shu-Ling Wang and Sunny S. J. Lin describes the most significant factors for personal, behavioral, and environmental influences in the social cognitive model of selfregulated learning. This model suggested, was applied to the development of the NetPorts web-based learning system. NetPorts reveals the interactions between these factors. Results of this attempt outlines the impact of the social cognitive view on self-regulated learning: students who are highly motivated, apply more effective strategies, and respond more properly to environmental demands, in the 
Web-based learning environment. These findings also further validate the application of the social cognitive model to Web-based learning through the NetPorts [30].

Anne Mendenhall \&Tristan E. Johnson present the social annotation model learning system (SAM-LS) designed in order to enhance student's critical thinking, critical writing and related literacy. And it is based in these four mechanisms(1) examples, (2) practice, (3) reflection and (4) collaboration. The Social Annotation Model uses HyLighter, an online annotation system that combines reading and writing, facilitates shared annotation practices and integrates annotations from multiple reviewers. This article reports on three studies held using HyLighter. In the first study, it was examined how students react in the learning environment, using HyLighter, The findings indicated that users' experiences were affirmative. The second study looked at students' ability to critically analyze information and reading comprehension using HyLighter in collaborative activities. Initial findings show that working in small collaborative groups may promote deeper thinking through peer interactions. The results of the third study indicated changes in reading comprehension, critical thinking and meta-cognition skills from the use of SAM-LS instructional strategies [31].

Piek Vossen presents the EuroWordNet project, based on WordNet, the on line English Thesaurus and lexical database which creates wordnets for other languages of Europe. The initial four languages are Dutch ,Italian, Spanish and English (later Czech, Estonian, German, and French will be added). Like the original Princeton WordNet, the new wordnets are hierarchies in which each node is a synset: a word sense, with which one or more synonymous words or phrases is associated. The synsets are connected by relations such as hyponymy, meronymy, and antonymy. However, some improvements have been made to the original design of WordNet. New relationships, including relationships across parts of speech, have been introduced [32].

\subsection{Mobile Applications}

Stacy Delacruz in her study presented the use of an application called Nearpod during guided reading instruction. Students and teachers who used this application reported that this method of guided reading was very effective in the comprehension of the text. Indeed, Nearpod turned out to be very helpful tool because of the userfriendliness, the ability to engage students and the ability of monitoring their effort [33].

Joerg Zumbach in his paper, raises the role of graphical and text argumentation tools in hypermedia learning. Specifically, the impact of visualization tools on argumentation skills, knowledge acquisition and motivation during learning with hypermedia learning environment are explored. In the experiment held, subjects had to complete an argumentation task using hypermedia learning environment as course. Some of the participants were given a graphical mind mapping tool whereas some others a two-columned text. A control group was given no argumentation task and no corresponding support device. Results revealed that the application of the argumentation task to hypermedia learning environments is a very effective method and developed knowledge acquisition [34]. 
In the same scientific filed Cameron Fadjo, Benjamin Friedman, John Black, Lettecha Johnson, investigate how a touch based gestural interface can have a positive effect in reading comprehension skills. In our study 16 second-grade students from two low-resource, high-needs urban public schools participated in an afterschool program where they physically interacted with and manipulated narrative elements (objects and characters) of critical sentences on an iPad. The results suggest that when elementary school children directly form characters and objects to create events while reading critical sentences (versus listening to a narrator read the same sentences) certain reading comprehension skills is enhanced [35].

In his paper, Antonio C. Encarnacion considers how hypertext - in its current nodeand-link form - can be enhanced by an adaptive, user-model-driven tool. During the processing of a document, the reader has to adapt to that document if the detail level is wrong the reader either skims the document or has to find additional sources of information for interpretation. The MetaDoc system not only has hypertext capabilities but also has knowledge about the documents it represents. Based on this knowledge the level of the document's presentation is modified in order to suit the user. MetaDoc builds and dynamically maintains a user model for each reader. The model connects the presentation of the document to the reader. MetaDoc is more a documentation reading system rather than a hypertext navigation or reading tool [36].

Speech-Reception-Threshold (SRT) tests measure an individual's ability to comprehend speech as far as concerns the influence of noise as well as the cognitive capacities. The paper of Sophia Cramer, Adriana A. Zekveld and Tammo Houtgast investigates the value of the Text-Reception-Threshold (TRT) test (a visual parallel of the SRT test) to assess the cognitive capacities allocated during speech comprehension. The relation between age, TRT, working memory (Spatial Span) and SRT were examined. The TRT and SRT correlated significantly, enhancing the value of TRT in explaining inter-individual differences in SRTs. The relations between age and TRT and between SSP and TRT were non-significant. The results outline that the current TRT test does not fully connect the cognitive aspects relevant in speech comprehension [37].

\subsection{Artificial Intelligence Applications}

In the research of it is supported that a new and more powerful type of computerassisted instruction (CAI), based on extensive application of artificial-intelligence (AI) techniques, is beneficial, and to demonstrate some of its major capabilities. A sum of computer programs was written and given the name SCHOLAR. The data base consists of structures of specific pieces of text, questions, and anticipated answers entered in advance by the teacher. By contrast, an information-structureoriented (ISO) CAI system is based on the usage of an information network of facts, concepts, and processes. It can generate text, questions, and corresponding answers. Because an ISO CAI system can also utilize its information network to answer questions generated by the student, a mixed-initiative dialogue between student and computer is possible with questions and answers from both sides [38] 
Christopher K. Riesbeck and Roger C. Schank present ELI (English Language Interpreter), a natural language parsing program currently used by several story understanding systems. ELI produces meaning representations (using Schank's Conceptual Dependency system) rather than syntactic structures. It also uses syntactic information only when the meaning can not be obtained directly and it talks to other programs that make high level inferences that tie individual events into coherent episodes. It makes use of context-based exceptions (conceptual and syntactic) to control its parsing routines[39].

\section{Discussion}

After all, perception of text is a matter of perception? What are the cognitive components which affect the comprehension process of the text? What are the characteristics of the text which are responsible for the communication success between the recipient and the transmitter of the textual message? Does perception play a significant role in this cognitive process? Which are the cognitive skills that make someone robust in text comprehension? Which are the contributions of ICTs in the assessment and training of the perception skill?

The purpose of this paper is to offer a comprehensive review on the current research approaches that focus on the relation of cognitive perception and text comprehension, the correlation of perception and the linguistic field of pragmatics as well as the contributions of ICTs in assessment and training of text comprehension.

The findings of this effort encompass the main cognitive aspects of perception that may affect text comprehension. Human brain creates situation models in order to comprehend a narrative text. Likewise, mental models turned out to contribute to foregrounding during text comprehension. It was also investigated the function of conscious and non conscious perception of text as there has been an approach towards the relation between phenomena experience and perceptual processes. Prior Knowledge plays also a significant role in cognitive text process as well as the event synthesis in human mind contributes to the organization of narrative understanding. Two other equally basic factors are causal inferences made in a text and the coherence relations with the linguistic markers in textual comprehension. Last but not least, emotional perception and reader's motivation when it comes to text comprehension are most determining in this procedure.

In the second part of this paper, there has been an attempt to examine the text perception through the point of view of pragmatics. More specifically, point-driven understanding may have an impact in the whole perception of a text. The making of irony is also a factor that can affect textual perception and of course the decoding of text connotation may lead to a certain text comprehension. There are also illustrated the pragmatic inferences related to logical terms, the function of moral text comprehension and how gestalt theory may function in the cognitive process of text understanding.

Regarding the role of ICTs in the field of cognitive and learning sciences, this paper offers a descriptive account of the web based technologies, mobile tools as well as 
Artificial Intelligence applications which enhance the assessment, training and evaluation of text comprehension. Firstly, it is exposed a study in which are investigated the effects of multimedia reading software on reading comprehension. There is also a description of the Reader Self Perception Scale, a new tool which measures how children fell about themselves as readers. There is also another on line test tool that determines the Common European Framework of Reference for Languages (CEFR) reading comprehension. Continually, Wen Hao Huang demonstrated findings of an evaluation of an on line game-based learning environment by examining the learners' motivational and cognitive processing. The following study illuminates the interaction between prior knowledge and concept mapping structure on hypertext comprehension. Accordingly, it is also investigated the nature of reading comprehension processes while reading on the Internet. The web based application called $3 D$ Readers, was used to train twenty middle school poor reading comprehenders in two meta cognitive strategies. NetPorts reveals the interaction among personal, behavioral, and environmental influences in the social cognitive model of self regulated learning. \&Tristan E. Johnson present the social annotation model learning system (SAM-LS) designed in order to enhance student's critical thinking, critical writing and related literacy. Lastly, Piek Vossen presents the EuroWordNet project.

As far as concerns the mobile applications, Nearpod is one of the tools used in elementary guided reading groups. It is also outlined that graphical and text based argumentation tools in hypermedia learning gains ground in the field text comprehension. The use of a touch-based interactive mobile App can improve text understanding. There is also a demonstration of the Metadoc system, as well as the SpeechReception-Threshold (SRT) tests which measure an individual's ability to comprehend speech.

And last but not least there was an attempt to describe the Artificial Intelligence applications such as CAI and ELI which provide with text comprehension processes.

Undoubtedly, text comprehension seems to be a more complex cognitive process. After all, human thought is a very complex existence. So, what can we say about perception of a text? It is logical elaboration, is it an emotional perception or an externalization of our social status? Surely, we have to admit that perception of a text has to do with our own personality, our experiences, ideals beliefs and ethics. Nevertheless, this paper gives motive for further research considerations.

\section{References}

[1] https://www.ncbi.nlm.nih.gov/books/NBK234157

[2] https://en.wikipedia.org/wiki/Perception

[3] https://en.wikipedia.org/wiki/Pragmatics

[4] 'Situation models, mental stimulations and abstract concepts in discourse comprehension'. Rolf A. Zwaan, Psychonomic Bulletin \& Review August 2016, Volume 23, Issue 4, pp $1028-1034$

[5] 'Accessibility and situation models in narrative comprehension' Daniel G. Morrow, Steven L. Greenspan, Gordon H. Bower, Journal of Memory and Language, Vol.26, issue 2, April 1987, pages 165-187 
[6] 'The construction of situation models in narrative comprehension. An event-indexing model' .Rolf A. Zwaan, Mark C. Langston, Arthur C. Graesser. Sage Journals, Vol.6 issue 5,Septemper 1995

[7] 'Mental models contribute to foregrounding during text comprehension'. Arthur M Glenberg, Marion Meyer, Karen Lindem, Journal of Memory and Language, Vol.26, issue 1, February 1987, pp 69-83

[8] 'Conscious and unconscious perception. An approach to the relation between phenomenal experience and perceptual processes'. Anthony J. Marcel, Cognitive Psychology, Vol.15, issue 2, April 1983, pp 238-300

[9] 'Conscious and non conscious perception. Experiments on visual masking and word recognition'. Anthony J. Marcel, Cognitive Psychology, Vol 15, issue 2, April 1983, PP197-237

[10] 'Learning form texts. Effects of prior knowledge and text coherence'. Danielle S. McNamara, Walter Kintsch, Journal of Discourse Processes, Vol 22, issue 3, 1996

[11] 'Prominent characters and events organize narrative understanding'. Daniel G. Morrow, Journal of Memory and Language, Vol24, issue 3, June 1985, pp304-319

[12] 'Causal inferences and the comprehension of narrative texts'. Paul Van den Broek, Psychology of learning and motivation, Vol.25,1990, pp 174-196

[13] 'Emotional perception of fairy tales. Achieving agreement in emotion annotation of text'. Ekaterina P. Volkova, Betty J. Mohle, Detmar Meurers, Dale Gerdemann, Heinrich H. Bulthoff, Proceedings of the NAACL HLT 2010 Workshop on Computational Approaches to Analysis and Generation of Emotion in Text, pages 98-106, Los Angeles, California, June 2010. c 2010 Association for Computational Linguistics

[14] 'The role of coherence relations and their linguistic markers in text processing'. Ted J. M. Sanders \& Leo G. M. Noordman, Discourse Processes, Vol29, issue 1, 2000

[15] 'Interest, learning and motivation', Ulrich Schiefele, Educational Psychologist, Vol26, issue $3-4,1991$

[16] 'Point driven understanding. Pragmatic and cognitive dimensions of literary reading'. Douglas Vipont, Russell A. Hunt, Poetics, Vol23, issue 3, June 1984, pp261-277

[17] 'Type of evaluation and making of irony. The role of perceived complexity and comprehension'. Christian Burgers, Margotvan, Mulken, Peter Jan Schellens, Journal of Pragmatics, Volume 44, Issue 3, February 2012, Pages 231-242

[18] 'The pragmatics of connotation', Keith Allan, Journal of Pragmatics, Vol39, issue 6, June 2007, pp1047-1057

[19] 'Pragmatic inferences relate to logical terms', Ira A. Noveck, Experimental Pragmatics pp 301-321, Part of the Palgrave Studies in Pragmatics, Language and Cognition book series (PSPLC)

[20] 'Moral text comprehension. Implications for education and research'. Darcia Narvaez, Journal of moral Education, Vol30, issue1, 2001

[21] 'Gestalt imagery. A critical factor in language comprehension'. Nanci Bell, Annals of Dyslexia, January 1991, issue 1, pp 246-260

[22] 'To gloss or not to gloss". An investigation of reading comprehension on line'. Lara L Lomicka, Language Learning and Technology, January 1998, Vol 1, num2, pp41-50

[23] https://prezi.com/j0e-7bbda9cg/the-reader-self-perception-scale-rsps/

[24] 'On line test tool to determine the CEFR reading comprehension level of text'. Eric Velleman, Thea van der Geest, Procedia Computer Science, Vol 27, 2014, pp350-358 https://doi.org/10.1016/j.procs.2014.02.039 
[25] 'Evaluating learner's motivational and cognitive processing in an on line game based learning environment'. Wen Hao Huang, Computers in Human Behavior, Vol 27, issue 2, March 2011, pp 694-704

[26] 'Interaction between prior knowledge concept mapping structure on hypertext comprehension'. Franck Amadieu, André Tricot, Claudette Mariné. Interacting with Computers, Volume 22, Issue 2, 1 March 2010, Pages 88-97

[27] 'Exploring the online reading comprehension strategies used by sixth grade skilled readers to search for and locate information on the internet', Julie Coiro, Elisabeth Dobler, Reading Research Quarterly, Vol 42, issue 2, April/May/June 2007, pp 214-257

[28] A model of online reading engagement: Linking engagement navigation and performance in digital reading',Johannes Naumann, Computers 'in Human Behavior, Vol 53, December 2015, pp263-277

[29] 'Web-based training of metacognitive strategies for text comprehension: Focus on poor comprehenders', Mina C. Johnson-Glenberg, Waisman Center University of Wisconsin Madison, WI, USA; The NeuronFarm, LLC Madison, WI, USA, Reading and Writing (2005) 18:755-786

[30] 'The application of social cognitive theory to web-based learning through NetPorts', ShuLing Wang and Sunny S. J. Lin, British Journal of Educational Technology Vol 38 No 4 2007 600-612 https://doi.org/10.1111/j.1467-8535.2006.00645.x

[31] 'Fostering the development of critical thinking skills, and reading comprehension of undergraduates using a Web 2.0 tool coupled with a learning system', Anne Mendenhall \&Tristan E. Johnson, Interactive Learning Environments, Vol 18, Issue3, 2010, pp 263276

[32] 'EuroWordNet: A Multilingual Database with Lexical Semantic Network', Piek Vossen, University of Amsterdam, Computational Linguistics Volume 25, Number 4, [Reprinted from Computers and the Humanities, 32(2-3), 1998] Dordrecht: Kluwer Academic Publishers, 1998, $179 \mathrm{pp}$; hardbound

[33] 'Using Nearpod in elementary guided reading groups'. Stacy Delacruz, Tech Trends, September 2014, volume 58, issue 5, pp62-69

[34] 'The role of graphical and text based argumentation tools in hypermedia". Joerg Zumbach, Computers in human Behavior, Vol 24, issue 4, Jule 2009, pp 811-817

[35] Improving reading comprehension skills using a touch-based interactive mobile app',Cameron Fadjio, Benjamin Friedman, John Black, Lettecha Johnson, Society for Information Technology and Teacher Education International Conference, Mar 2012, Austin, Texas,USA

[36] 'Metadoc: An Adaptive Hypertext Reading System', Craig Boyle Information Engineering, P.O. Box 18002/, Austin, Texas 78718, USA; e-mail: boyle@cactus.org, Antonio O. Encarnacion,Department of Computer Science, Texas A\&M University, College Station, Texas 77843, USA, Brusilovsky et al. (eds.), Adaptive Hypertext and Hypermedia, 71-89, 1994

[37] 'Measuring cognitive factors in speech comprehension: The value of using the Text Reception Threshold test as a visual equivalent of the SRT tes', Sophia Cramer, Adriana A. Zekveld and Tammo Houtgast, Scandinavian Journal of Psychology, Volume 50, Issue 5October 2009 Pages 507-515

[38] 'AI in CAI: An Artificial-Intelligence Approach to Computer-Assisted Instruction', Jaime R. Carbonell, IEEE Transactions on Man-Machine Systems ( Volume: 11, Issue: 4, Dec. 1970 ),pp190-202

[39] 'Comprehension by Computer: Expectation-Based Analysis of Sentences in Context.', Christopher K. Riesbeck, Roger C. Schank, Oct. 1976, pp82 


\section{$7 \quad$ Authors}

Aglaia Tourimpampa is with N.C.S.R. 'Demokritos', Institute of Informatics and Telecommunications, Telecoms Lab - Net Media Lab, Agia Paraskevi, 153 10, Athens, Greece (e-mail: lidatou@gmail.com).

Athanasios Drigas is a Senior Researcher at N.C.S.R. Demokritos. He is the Coordinator of Telecoms Lab and founder of Net Media Lab since 1996. From 1985 to 1999 he was the Operational manager of the Greek Academic network. He has been the Coordinator of Several International Projects, in the fields of ICTs, and e-services (elearning, e-psychology, e-government, e-inclusion, eculture etc). He has published more than 280 articles, 7 books, 25 educational CD-ROMs and several patents. He has been a member of several International committees for the design and coordination of Network and ICT activities and of international conferences and journals. (email: dr@iit.demokritos.gr).

Alexandra Economou is an associate professor at the University of Athens in the department of Psychology. She teaches undergraduate and postgraduate neuropsychology and experimental psychology courses at the University of Athens in the Department of Psychology while her interests focus on cognitive and biological psychology. Dr. Economou is also vice-president of the Greek Aphasia Association (E-mail: aoikono@psych.uoa.gr).

Petros Roussos is an assistant professor at the University of Athens in the department of Psychology. Since 1994 he has taught cognitive psychology, research methods and statistics in psychology at the University of Crete (Greece), the University of the Aegean (Greece), the University of Athens and many other colleges and higher education institutions. He has served as adjunct research fellow of the Hellenic Pedagogical Institute (1998-2000), as Psychology Department Chair at the University of Indianapolis Athens Campus (2000-2004) and as Researcher at the Unit of Analysis and Documentation at the Greek Ministry of Employment and Social Protection (2004-2005). He is also a graduate member of the British Psychological Society, member of the Hellenic Psychological Society, and General Secretary of the Hellenic Cognitive Science Society (E-mail: roussosp@psych.uoa.gr).

Article submitted 30 October 2017. Resubmitted 25 April 2018. Final acceptance 07 May 2018. Final version published as submitted by the authors. 